\title{
Diseño de Equipo para la Separación Primaria de los Sólidos de las Aguas Residuales en las Empresas Porcinas en Cuba
}

\section{Equipment Design for the Primary Separation of Solids from Wastewater in Swine Companies in Cuba}

DOI: https://doi.org/10.17981/ijmsor.04.01.07

\author{
Research Article - Reception Date: Apr 29, 2019- Acceptance Date: Aug 17, 2019 \\ Rafael Antonio Goytisolo Espinosa \\ Universidad "Carlos Rafael Rodríguez". Cienfuegos (Cuba) \\ ragoyti@ucf.edu.cu \\ Juan José Cabello Eras \\ Universidad de la Costa. Barranquilla (Colombia) \\ jcabello2@cuc.edu.co
}

To reference this paper:

R. Goytisolo Espinosa \& J. Cabello Eras, "Diseño de equipo para la separación primaria de los sólidos de las aguas residuales en las empresas porcinas en Cuba", IJMSOR, vol. 4, no. 1, 2019. https://doi. org/10.17981/ijmsor.04.01.07

Resumen-- En el Trabajo se profundiza en la literatura acerca de los aspectos que caracterizan la generación de residuales en las Granjas Porcinas en general y en particular las existentes en Cuba. Se revisaron algunos aspectos vinculados con el equipamiento empleado en la separación primaria de los sólidos de las aguas residuales de las Empresas Porcinas, para seleccionar el más adecuado desde el punto de vista de la capacidad necesaria, economía, sencillez de operación y mantenimiento y efectividad. Se selecciona en definitiva como equipo base para la separación un Transportador / Colador por Tornillo Sin Fin, se calcula la capacidad necesaria, sus dimensiones básicas y parámetros fundamentales de operación y se selecciona el moto reductor para su accionamiento. Finalmente se diseña y calcula el árbol del transportador utilizando el esquema de análisis de viga sobre fundación elástica y se diseñan y calculan los cojinetes empleando un material plástico FEROFOM.

Palabras clave-- Aguas residuales; empresas porcinas; residuos solidos

\begin{abstract}
In the Work the literature is deepened about the aspects that characterize the generation of residuals in the Pig Farms in general and in particular those that exist in Cuba. Some aspects related to the equipment used in the primary separation of the solids from the waste water of the Swine Companies were reviewed, to select the most appropriate from the point of view of the necessary capacity, economy, simplicity of operation and maintenance, and effectiveness. Ultimately, a Conveyor / Strainer by Endless Screw is selected as the base equipment for the separation, the necessary capacity, its basic dimensions and fundamental operating parameters are calculated and the reduction motor is selected for its operation. Finally, the conveyor shaft is designed and calculated using the elastic foundation beam analysis scheme and the bearings are designed and calculated using a FEROFOM plastic material.
\end{abstract}

Keywords-- Wastewater; pig companies; solid waste 


\section{INTRODUCCIÓN}

Este trabajo es realizado en la Empresa Porcina de Cienfuegos, la cual tiene como objeto social la producción y comercialización de cerdos para el mercado nacional. En la misma no se le está dando el uso adecuado a los desechos residuales ya que solamente se realiza un solo tratamiento que el uso de lagunas de sedimentación y fermentación. Estos residuos pueden ser utilizados como biogás, biofertilizante, como alimentos para otras especies de animales y para los propios cerdos, etc. En el trabajo se propone diseñar un equipo para la separación primaria de los sólidos de las aguas residuales del porcino para su aprovechamiento posterior.

\section{Desarrollo}

En la literatura se describen distintos sistemas para la separación líquido-sólido mediante el tamizado como son: transportadores de arrastre con malla para el tamizado, rastrillos como el empleado en la separación del bagacillo del guarapo en la industria azucarera y los transportadores de tornillo sinfín con malla tamizada en la parte inferior del cuerpo del transportador. Son muy baratos pero su efectividad en la separación es inferior a la de los filtros rotatorios al vació, sin embargo, en Cuba se logran excelentes resultados en la separación del bagacillo. Entre los equipos que se emplean en los procesos de tratamiento de los residuales se encuentran las bombas de cavidad progresiva, o también conocidas como bombas MONO. Estas bombas debido a su gran versatilidad, características y campo de aplicaciones que va desde líquidos tan ligeros y limpios como el agua, hasta líquidos tan pesados como las mieles y lodos conteniendo un gran número de partículas en suspensión las mismas se han difundido en casi todas las ramas industriales a nivel mundial. Estas bombas son fabricadas actualmente en Cuba [5] y pueden ser utilizadas para el bombeo de las aguas y los sólidos residuales de las Empresas Porcinas en Cuba.

En la empresa Porcina de Cienfuegos el único tratamiento que en la actualidad se les realiza a los residuos es exclusivamente el uso de uso de laguna de almacenaje y fermentación (laguna de oxidación). La empresa Porcina de Cienfuegos cuenta con dos Integrales Porcinos el I y el II, existiendo un multiplicador y una laguna de oxidación.

Según [2] en las granjas evaluadas en el Proyecto 7.2.56: Tratamiento de aguas en zonas industriales urbanas y rurales en México, el número total de cerdos que se albergan en la granja en las distintas áreas es de 352. De acuerdo con los datos relativos a la cantidad de agua que se consume por ingesta, y para el lavado de las unidades, se estima que el consumo de agua de

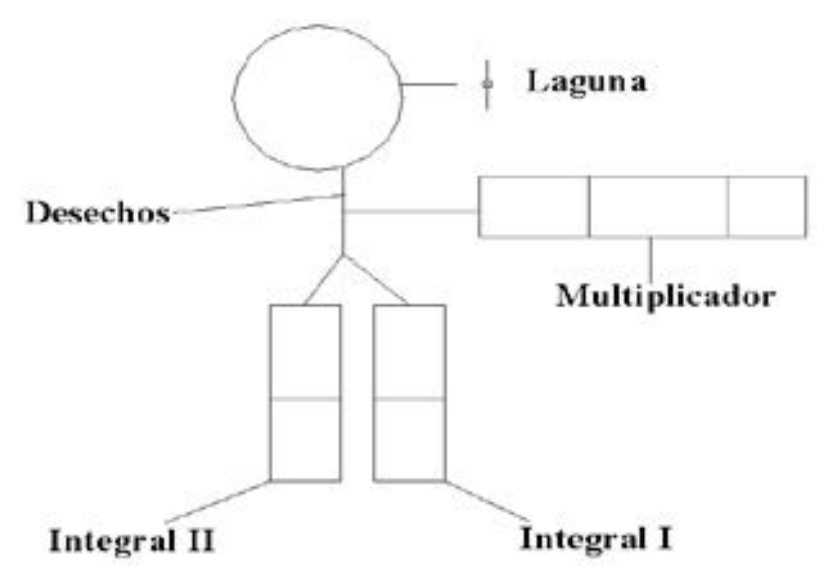

Fig. 1. Estructura de la Empresa Porcina de Cienfuegos

primer uso, en promedio semanal, es de $62.428 \mathrm{~m}^{3}$, volumen que genera $37.41 \mathrm{~m}^{3} / \mathrm{semana}$ de aguas residuales, considerando que el $15 \%$ del agua de ingesta retorna como orina. Estos datos suministrados por estos investigadores equivalen a aproximadamente a una generación de aguas residuales promedio de aproximadamente 16 litros/día/cerdo. Según estos autores, para el tratamiento de las aguas residuales las granjas cuentan con una planta constituida por los procesos físicos de eliminación manual de los sólidos de mayor tamaño (excretas), deshidratación (mediante un filtro rotatorio de baja presión) de los sólidos contenidos en las aguas residuales, y éstas sometidas a los procesos de sedimentación y filtración. Señalar además que por su experiencia los cerdos en crecimiento solamente utilizan entre el 30 y el 35\% del nitrógeno y el fósforo ingeridos, pues eliminan el remanente a través de las excretas, por lo que éstas poseen virtualmente todos los nutrimentos que requieren las plantas para su crecimiento y desarrollo, y representan un recurso valioso que puede reemplazar insumos costosos en la producción de forrajes y otro tipo de cultivos, de aquí la necesidad de su aprovechamiento [10]. Según [3] en Cuba existen centros integrales de ganado porcino con diferentes capacidades llegando hasta 25.000 cabezas. En la alimentación de los mismos se utiliza miel, pienso líquido (alimento elaborado) y pienso seco; la limpieza de las naves se efectúa con agua a presión, en dependencia de muchos factores (presión de agua, salideros, tiempo de limpieza y otros). El residual porcino es el agua que se recoge de los centros integrales, sin previa separación de sólidos por tamizado o decantación. El volumen de vertido puede oscilar entre 60 y 80 litros por puerco de $100 \mathrm{~kg}$ de peso; su característica principal es el importante contenido en sólidos totales, de 25 a $50 \mathrm{~kg} / \mathrm{m}^{3}$, de los que un $80 \%$ son sólidos en suspensión, y hasta un $30 \%$ de la materia seca son sólidos minerales. La composición de este residual está relacionada con la alimentación de los animales y con la inclusión de productos que inducen el crecimiento acelerado, medicamentos, metales pesados (zinc y cobre), cuya persistencia en los residuos puede ocasionar daños imprevisibles en su utilización y posterior aplicación. 
El manejo de los residuales porcinos resulta complejo. El tratamiento de los residuales porcinos con estos sistemas permite la disminución de la carga orgánica del residual, por tanto, disminuyen la contaminación de ríos, lagos y mares, lo que contribuye al cuidado del medio ambiente y de los ecosistemas aledaños a las instalaciones.

En la Empresa Porcina de Cienfuegos se llega a tener una capacidad de hasta 25.000 cabezas lo que provoca una elevada contaminación. La alimentación de los mismos depende de las características de los centros utilizándose miel, pienso líquido (alimento elaborado) y pienso seco fundamentalmente. La limpieza de las naves se efectúa con agua a presión reportándose valores de 20 - 80 l./puerco-día, en dependencia de muchos factores (presión de agua, salideros, tiempo de limpieza y otros). Las características de las aguas residuales han sido publicadas, encontrándose los principales parámetros dentro de los siguientes valores: Sólidos Totales (11 - 20 g/l), Sólidos Volátiles Totales (70 - 77\% ST), Sólidos Suspendidos Totales (7.7 - 14.2 g/l), Demanda Química de Oxígeno, DQO (17.3 - 25.4 g/l), Demanda Bioquímica de Oxígeno, DBO (7.8 - 12.4 g/l), $\mathrm{pH}$ (6.4 - 7.1). Considerando la presencia de 25.000 puercos por Granja Integral Porcina y un consumo de agua total para limpieza e ingesta de $80 \mathrm{l} /$ puerco/día y una cantidad máxima de sólidos, de $50 \mathrm{~kg} / \mathrm{m}^{3}$, se obtiene que la cantidad máxima de sólidos a separar de esos residuales líquidos se corresponde con:

$$
\mathrm{Q}=25000 \cdot 80 \cdot 50 / 24 \cdot 1000=4167 \mathrm{~kg} / \mathrm{h}
$$

Teniendo en cuenta que esta cifra se corresponde con las condiciones extremas, se considerará como capacidad máxima del Transportador-Colador Sin Fin a diseñar la de $Q=4000 \mathrm{~kg} / \mathrm{h}$. En la Fig. 2 se muestra un esquema con el diseño conceptual del Transportador-Colador Sin Fin a diseñar.

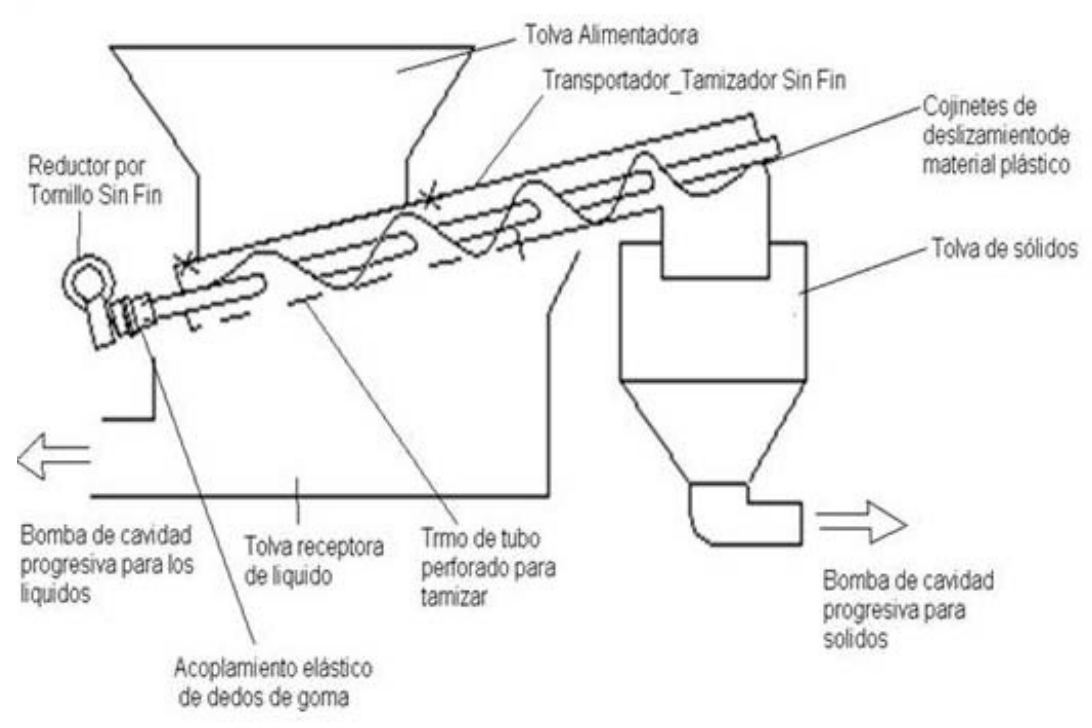

Fig. 2. Esquema del Transportador - Colador de Tornillo sin fin a diseñar.
El peso volumétrico del material a transportar fue obtenido mediante el pesaje de varias muestras de $1.000 \mathrm{~cm}^{3}$ en las condiciones de humedad en que el mismo es transportado, obteniéndose, como valor medio: $\gamma=1.198 \mathrm{gr} / \mathrm{cm}^{3}=1.200 \mathrm{~kg} / \mathrm{m}^{3}$. La capacidad de transportación del sinfín de fondo se puede calcular por la expresión Labahn-Kaminsky [4], [8]:

$$
\mathrm{Q}=60 / 4(\mathrm{D} 2-\mathrm{d} 2) \mathrm{s} \cdot \mathrm{n} \cdot \mathrm{f} \cdot \gamma \cdot \mathrm{CB} \quad \mathrm{kg} / \mathrm{h}
$$

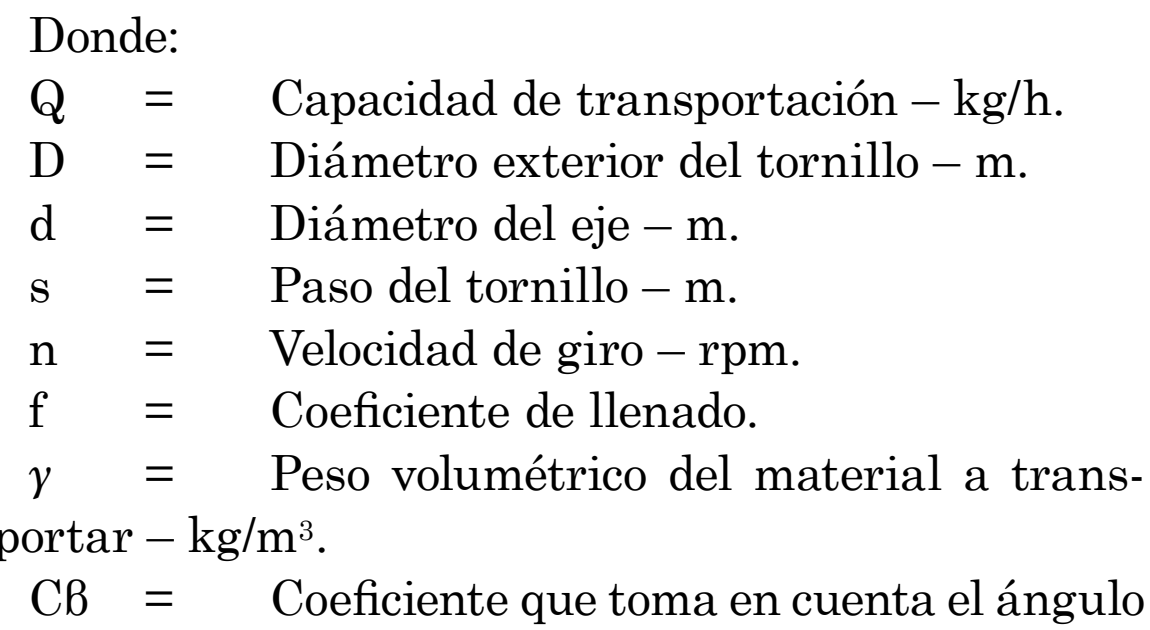
de inclinación del tornillo.

Según [8] para materiales pesados, abrasivos como: cemento, arena, etc.la velocidad máxima del sinfín puede alcanzar valores de:

$$
n_{\max }=\frac{30}{\sqrt{D}}
$$

Teniendo en cuenta lo agresivos que son los residuales porcinos se utilizará esta última recomendación de manera que la velocidad de giro del tornillo sea más bien lenta, eso contribuirá por otro lado a que exista el tiempo necesario para que el líquido se escurra. El coeficiente de llenado del transportador se recomienda elegirlo, en función del material a transportar, entre $\mathrm{f}=0,125-0,4$, los valores mayores para materiales no abrasivos. En el caso analizado de un transportador-colador, teniendo en cuenta la presencia de una mezcla líquido-sólido en el mismo, lo que implica que no se llenará nunca completamente, este coeficiente se tomará como un valor medio, o sea, $\mathrm{f}=0,25$. El coeficiente $\mathrm{C} 6$ se extrapoló de los valores que aparecen en la referencia [8] obteniéndose para $\beta=30^{\circ}, C \beta=0,5$. Considerando tentativamente, para poder estimar el diámetro exterior (D) del transportador la siguiente expresión aproximada para la capacidad del mismo:

$$
\begin{aligned}
& Q=\frac{60 \pi}{4} \cdot D^{2} \cdot s \cdot n \cdot f \cdot \gamma \cdot C_{\beta} \\
& \frac{D^{2}}{\sqrt{D}}=\frac{4 Q}{60 \pi \cdot s \cdot 30 \cdot f \cdot \gamma \cdot C_{\beta}}
\end{aligned}
$$


De donde despejando D y sustituyendo, se obtiene que: $\mathrm{D}=0,196 \mathrm{~m}$. Se tomará $\mathrm{D}=0,225 \mathrm{~m}$ para tomar en cuenta la reducción del área efectiva por la presencia del espacio ocupado por el diámetro del árbol en la expresión que no se consideró en la expresión de capacidad utilizada. Se asume tentativamente, teniendo en cuenta que el árbol es largo $d=6 \mathrm{~cm}=0,06 \mathrm{~m}$. Se recalculará entonces la capacidad real.

$Q=\frac{60 \pi}{4} \cdot\left(0,225^{2}-0,06^{2}\right) \cdot 0,2 \cdot \frac{30}{\sqrt{0,25}} \cdot 0,25 \cdot 1200 \cdot 0,5=3989 \mathrm{~kg} / \mathrm{h} \approx 4000 \mathrm{~kg} / \mathrm{h}$.

La velocidad de giro requerida en el eje del transportador será: $\mathrm{n}=63,3 \mathrm{rpm}$. La potencia requerida se calculó por la ecuación dada por [8]:

$$
N_{\mathrm{t}}=\mathrm{Q} \cdot \mathrm{L} / 367(\mathrm{w}+\operatorname{sen} \beta) \mathrm{kW}
$$

$\mathrm{Q}=$ Capacidad del transportador en $\mathrm{t} / \mathrm{h}$.,

$\mathrm{L}=$ Longitud del transportador en $\mathrm{m}$,

$\mathrm{W}=$ Coeficiente empírico de resistencia al movimiento, que depende del tipo de material a transportar.

La magnitud se toma:

2,5 = Para antracita, carbón pardo, sal en piedras.

$4=$ Para yeso, arcilla, cemento, ceniza, arena, caliza.

$\beta=\quad$ Ángulo de inclinación del transportador.

Calculando para los datos del Transportador-Colador, considerando $\mathrm{w}=4$.

$$
\mathrm{N}_{\mathrm{t}}=(4.3 / 367)\left(4+\operatorname{sen} 30^{\circ}\right)=0,15 \quad \mathrm{~kW}
$$

La transmisión en este caso está compuesta por un reductor sinfín de simple paso, simple hélice. Se asumió $\eta r e d=0,7$. De donde, la potencia requerida en el motor:

$$
\mathrm{Nm}=0,15 / 0,7=0,22 \mathrm{~kW}
$$

Se selecciona un moto-reductor sinfín de una potencia igual a $\mathrm{N}=0,25 \mathrm{~kW}$, una velocidad de giro del motor $\mathrm{n}=1200 \mathrm{rpm}$ y una relación de transmisión del reductor sinfín de $\mathrm{i}=20$, lo que garantizaría una velocidad de giro del tornillo de $\mathrm{n}_{\mathrm{t}}=60 \mathrm{rpm}$, que es la deseada. El árbol del transportador es en la realidad una viga sobre fundación elástica. El mismo tiene dos apoyos articulados en los extremos, pero posee una gran longitud, por lo que su peso propio distribuido q tenderá a provocar una deflexión que provocará que tienda a apoyarse en primer lugar en el propio producto que transporta y en segundo lugar en la propia hélice del tornillo. Es muy difícil precisar donde existirá mayor tendencia a apoyarse, no obstante, se elegirá este esquema para calcular el árbol del transportador pues se considera más exacto la imprecisión podrá estar en el coeficiente de rigidez de la fundación el cual puede ser determinado experimentalmente, pero en este trabajo se estimará en función de experiencias de aplicación de este esquema en otros casos prácticos. En la Fig. 3 se muestra el esquema utilizado.

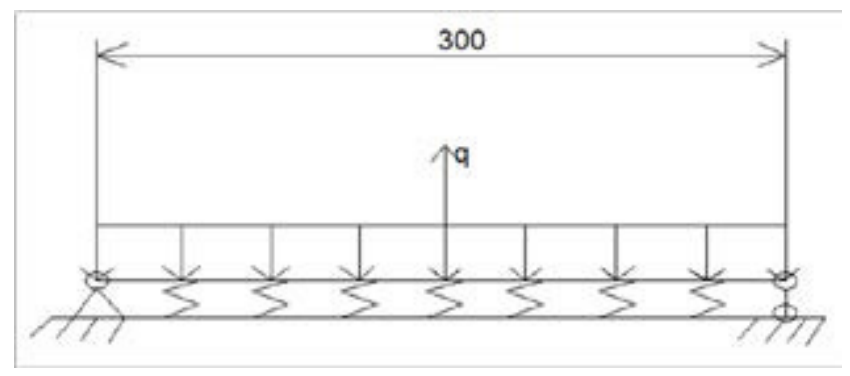

Fig. 3. Esquema de Análisis propuesto para el árbol del transportador de Tornillo.

Para aplicar el esquema de análisis de viga sobre fundación elástica se tiene que encontrar el valor para el coeficiente de rigidez de la fundación, a, de la mezcla residuales sólidos-líquidos. En la literatura no aparece ningún valor para este parámetro en las condiciones de apoyo sobre esta mezcla. En la investigación realizada en el trabajo [6] sobre la consideración de los tornillos helicoidales de las bombas MONO, los cuales están apoyados en una base elástica (los estatores de goma), se demostró que se podía utilizar un valor del coeficiente de rigidez de la fundación igual a: $\alpha=63,64 \mathrm{MPa}$. Este valor es para el caso del contacto goma-acero, para el caso caña húmeda triturada y compactada-hierro fundido en el trabajo [1] se obtuvo experimentalmente un valor $\alpha=8000 \mathrm{MPa}$, valor este muy elevado a causa de la presencia del agua, pero en este caso la goma es por mucho más elástica. En el trabajo [9] para el contacto acero - cemento, se utilizó $\alpha=230 \mathrm{MPa}$. En esta ocasión que no se conoce nada sobre el coeficiente de balastro de la mezcla residual y la imposibilidad por falta de tiempo de realizar algún experimento, se asumirá un valor en función de estas experiencias anteriores. Teniendo en cuenta que la mezcla es blanda, pero tiene un alto contenido de agua la cual es incompresible, se tomará un valor de a sólo un poco más elevado que el contacto gomaacero, aunque no tan rígido como el contacto entre acero-cemento. Se propone emplear a $=80 \mathrm{MPa}$. Para calcular la carga q uniformemente distribuida sobre el árbol se considerará lo siguiente:

Dicha carga está compuesta por el peso del árbol y por el peso de la hélice del tornillo. 
El peso del árbol de $\mathrm{d}=6 \mathrm{~cm}$ y $\mathrm{L}=300 \mathrm{~cm}$ de acero inoxidable es $66 \mathrm{~kg}$.

El peso de la hélice, teniendo en cuenta que su paso es $\mathrm{s}=20 \mathrm{~cm}$ y que por lo tanto con la longitud $\mathrm{L}=300$ $\mathrm{cm}$ y caben 15 discos de las siguientes dimensiones.

Los discos serán de espesor $\mathrm{t}=0,4 \mathrm{~cm}$.

El peso de un disco será: $\mathrm{W}=1,15 \mathrm{~kg}$.

El peso de los 15 discos que conformarán la hélice será: $\mathrm{Wd}=18 \mathrm{~kg}$.

El peso de la carga total sobre el árbol será: $\mathrm{W}_{\mathrm{a}}=66$ $+18=84 \mathrm{~kg}$.

y la carga distribuida: $\mathrm{q}=\mathrm{W}_{\mathrm{a}} / \mathrm{L}=84 / 300=0,28 \mathrm{~kg} /$ cm.

Para $d_{\mathrm{a}}=6 \mathrm{~cm}$

$$
\begin{gathered}
I_{\mathrm{a}}=0,1 * \mathrm{~d} 4_{\mathrm{a}}=0,1 * 64=129.6 \mathrm{~cm} 4 \\
\beta=\sqrt[4]{\frac{\alpha}{4 E I}}=\sqrt{\frac{8}{4 * 2,2 * 10^{4} * 129.6}} \cong 0,029 \mathrm{~cm}^{-1}
\end{gathered}
$$

Para $\mathrm{z}=300 \mathrm{~cm}$ el parámetro adimensional:

$$
\xi=\beta * Z=0,029 * 300=300=8.7
$$

Los parámetros de origen conocidos son: $Y_{0}=0, Y_{1}=$ $0, M_{0}=0, M_{1}=0$

$$
\begin{aligned}
& Y_{l}=\frac{\theta_{0}}{0,029} * J_{2(8,7)}-\frac{1}{E I_{a}}\left[\frac{Q_{0}}{(0,029)^{3}} * J_{4(8,7)}\right]+\frac{1}{4(0,029)^{4}} * q\left[J_{1(8,7)}-J_{I(0)}\right]=0 \\
& M_{l}=\frac{Q_{0}}{0,029} * J_{2(8,7)}+\frac{8}{(0,029)^{3}} * \theta_{0} * J_{4(8,7)}+\frac{1}{(0,029)^{2}} * q\left[J_{3(8,7)}-J_{3(0)}\right]=0
\end{aligned}
$$

Interpolando de la Tabla de las Funciones de Krilov [7]:

Tabla 1. Interpolación funciones de Krilov para Z $=300$ CM

\begin{tabular}{|l|l|l|l|l|}
\hline$\xi=8 * Z$ & \multicolumn{1}{|c|}{$\mathrm{J} 1(\xi)$} & \multicolumn{1}{|c|}{$\mathrm{J} 2(\xi)$} & \multicolumn{1}{|c|}{$\mathrm{J} 3(\xi)$} & \multicolumn{1}{c|}{$\mathrm{J} 4(\xi)$} \\
\hline 8,5 & -1470.37 & -241.41 & 981.1 & 860.39 \\
\hline 8,7 & -2364.37 & -548.998 & 922.604 & 1052.354 \\
\hline 9,0 & -3691.48 & -1010.38 & 834.86 & 1340.3 \\
\hline
\end{tabular}

Se obtiene: $Q O=0,06 \theta_{0}=-5,44+10^{-6} \mathrm{rad}$

El momento flector máximo en el árbol se producirá para $Z=150 \mathrm{~cm}$ :

TABla 2. Interpolación FunCiONES DE KRILOV PARA Z $=150 \mathrm{CM}$

\begin{tabular}{|l|c|c|c|c|}
\hline$\xi=8 * \mathrm{Z}$ & $\mathrm{J} 1(\xi)$ & $\mathrm{J} 2(\xi)$ & $\mathrm{J} 3(\xi)$ & $\mathrm{J} 4(\xi)$ \\
\hline 4,30 & $-14,7722$ & -24.2669 & -16.8773 & $-4,7501$ \\
\hline 4,35 & -13.6451 & -24.9521 & -19.1258 & -5.6558 \\
\hline 4,40 & -12.5180 & -25.6373 & -19.3743 & -6.5615 \\
\hline
\end{tabular}

Calculando el Momento Flector en la mitad de la longitud del árbol:

$$
\begin{aligned}
& M_{(150)}=\frac{Q_{0}}{0,029} * J_{2(4,3 s)}+\frac{8}{(0,029)^{2}} * \theta_{0} * J_{4(1+3, s)}+\frac{1}{(0,029)^{2}} * q\left[J_{3(3,3)}-J_{3(0)}\right] \\
& M_{(\mathbf{s i d})}=\frac{0,06}{0,029} *(-24,95)+\frac{8}{(0,029)^{3}} *\left(-5,44 * 10^{-6}\right) *(-5,6558)+\frac{1}{(0,029)^{*}} * 2,747 * 10^{-*} *
\end{aligned}
$$

$$
M_{(150)}=-100,73 \mathrm{kN}-\mathrm{cm}
$$

La tensión de flexión será:

$$
\sigma=M_{\mathrm{f}} / \mathrm{W}=100.73 / 43,2=2,39 \mathrm{kN} / \mathrm{cm} 2
$$

En la Tabla 3 se dan las propiedades del acero inoxidable AISI 316 en barras en estado normalizado y los factores de seguridad del árbol con relación a la fluencia. Como se puede apreciar el factor de seguridad con relación a la fluencia es $n=8,6$, lo que se considera elevado. El árbol no debe tener problemas con la resistencia.

Para la construcción de los apoyos del Equipo para la Separación de los Residuales Sólidos de la Empresa Porcina de Cienfuegos se propone utilizar cojinetes de deslizamiento de material plástico fabricados por TENMAT. Este es un importante fabricante, especializado en materiales y componentes industriales no metálicos de alto rendimiento, suministrados en un amplio rango de aplicaciones industriales en todo el mundo. TENMAT tiene su sede central en Strafford Park, Manchester, con filiales en el Reino Unido

Tabla 3. Propiedades del acero inoxidable AISI - 316, Factor De SEguridad obTenido.

\begin{tabular}{|c|c|l|c|c|}
\hline $\begin{array}{c}\text { Límite de } \\
\text { fluencia }\end{array}$ & \multicolumn{1}{|c|}{$\begin{array}{c}\text { Resistencia } \\
\text { máxima }\end{array}$} & $\begin{array}{c}\text { Módulo de } \\
\text { Elasticidad }\end{array}$ & $\begin{array}{c}\text { Tensión real } \\
\text { generada }\end{array}$ & $\begin{array}{c}\text { Factor de } \\
\text { seguridad }\end{array}$ \\
\hline of $=20,5 \mathrm{kN} / \mathrm{cm} 2$ & ou $=51,5 \mathrm{kN} / \mathrm{cm} 2$ & $\mathrm{x} \mathrm{Pa}$ & $\sigma \mathrm{u}=2,39 \mathrm{kN} / \mathrm{cm} 2$ & $n=\frac{\sigma_{f}}{\sigma}=\frac{20,5}{2,39}=8,6$ \\
\hline
\end{tabular}


(Rugby y Cradley Heath), Francia, Alemania, Italia y los Estados Unidos. Las dimensiones de los bujes, tolerancias, dilatación por efecto del agua y la dilatación térmica, así como las holguras con el árbol y el número y las dimensiones de las ranuras de lubricación se realizaron según las recomendaciones de los fabricantes (FEROFORM; Design, Machining and Installation Manual, 2011). La presión de Trabajo Máxima Normal especificada por el fabricante es $p_{\max }=65 \mathrm{MPa}=$ $6,5 \mathrm{kN} / \mathrm{cm}^{2}$, para el caso de los cojinetes diseñados, la fuerza normal es igual a la reacción $\mathrm{Qo}=0,06 \mathrm{kN} \mathrm{y}$ el área proyectada es $\mathrm{A}=\mathrm{L} \cdot \mathrm{d}=9 \cdot 6=54 \mathrm{~cm}^{2}$ y la presión de trabajo real para esta fuerza radial sobre el cojinete será: $p=0,06 / 54=0,0011 \mathrm{kN} / \mathrm{cm}^{2}$ muy por debajo de la máxima permisible. Es de esperar que el desgaste sea mínimo en el cojinete.

\section{CONCLUSIONES}

- Se realizó el Diseño Conceptual del Equipo a diseñar, Se precisó la capacidad necesaria para satisfacer una Granja Integral Porcina como la de Cienfuegos. Se calcularon los parámetros dimensionales del Transportador - Colador, su potencia y velocidad de giro y se seleccionó los su transmisión y el motor eléctrico.

- Se realizó el cálculo del árbol del transportador aplicando el Esquema de Análisis de Viga sobre Fundación Elástica y se calcularon y dimensionaron los cojinetes de deslizamiento fabricados de material plástico FEROFORM. Se evaluó la resistencia tanto del árbol como de los cojinetes, resultando ambas adecuadas.

\section{REFERENCIAS}

[1] N. Arzola, "Determinación Experimental del Coeficiente de Rigidez del Bagazo", Ing. Investig., vol. 17, no. 2, pp. 5-10, Ago, 2007. http://www.scielo.org.co/pdf/iei/v27n2/ v27n2a01.pdf

[2] J. De Victoria y M. Galván, "Tratamiento y aprovechamiento de aguas residuales de granjas porcinas, primera etapa", Proyecto 7.2.56. Tratamiento de aguas en zonas industriales urbanas y rurales. Ingeniería Ambiental. México. 2006.

[3] J. Morales, T. Barceló y H. Hernández Herrera, "Alternativas para la revalorización de los efluentes de la de la Empresa Porcina Cienfuegos", IJMSOR, vol. 2, no. 1, pp. 22-29, Dec. 2017. Recuperado de http://ijmsoridi.com/ index.php/ijmsor/article/view/83

[4] O. Labahn-Kaminsky, Prontuario del Cemento. La Habana: Ciencia y Técnica, 1970.

[5] Y. Llody, "Nuevos métodos de cálculo y tecnologías de fabricación de los rotores de las bombas de cavidad progresiva", Tesis Doctoral, Universidad de Cienfuegos, Cuba, 2011.

[6] D. López, "Nuevo esquema de análisis para el cálculo de las fuerzas internas y los desplazamientos en los rotores de las bombas de cavidad progresiva", Trabajo de Diploma. UCF, USA, 2009

[7] G. S. Pisarenko, A. P. Yakovlev y V. K. Matveev, Manual de Resistencia de Materiales. Moscú: Editorial MIR, 1989.

[8] V. S. Shubin y C. Pedré, Diseño de Maquinaria Industrial. La Habana, CU: Editorial Pueblo y Educación, 1984.

[9] A. Valle, "Diseño y Fabricación de Basculador de 20 t. sobre terreno", Tesis de Maestría, 2000.

[10] M. Bertolli, G. Roark, S. Urrutia y F. Chiodi, "Revisión de modelos de madurez en la medición del desempeño", INGE CUC, vol. 13, no. 1, pp. 70-83, jun. 2017. https://doi. org/10.17981/ingecuc.13.1.2017.07 